\section{Étude ethnobotanique et variabilité morphologique de Syzygium guineense var. macrocarpum dans les hautes savanes guinéennes de l'Adamaoua (Cameroun)}

Georges Maxime LAMY LAMY

\section{RÉSUMÉ}

La problématique de la variabilité morphologique des feuilles et fruits de nombreuses plantes locales multifonctionnelles préoccupe les chercheurs africains. À cela s'ajoute la menace de disparition de certaines de ces plantes vivant encore naturellement. Dans la zone agroécologique des hautes savanes guinéennes de l'Adamaoua au Cameroun se rencontre Syzygium guineense var. macrocarpum (Engl.) F. White, une myrtacée endémique. Pour sa domestication, un problème de choix des sous-variétés à retenir s'est posé. L'ethnobotanique étant la relation entre l'Homme et les plantes dans un milieu donné à travers le temps, la prise en compte du savoir et des préférences locales était indispensable. Par ailleurs, les objectifs visaient à identifier, décrire et déterminer les descripteurs morphologiques (qualitatifs et quantitatifs) et les sous-variétés de la plante. Les personnes enquêtées étaient constituées de 218 hommes et femmes appartenant à neuf ethnies différentes. Dans des quadrats de $100 \mathrm{~m} \times 100 \mathrm{~m}, 256$ arbres des sous-variétés de la plante ont été sélectionnés. Le dispositif expérimental était un plan factoriel $(8 \times 4 \times 8 \times 3): 8 \times 4$ pour l'unité expérimentale ; 8 pour le nombre de sites d'étude ; 4 pour le nombre de sous-variétés ; 3 pour le nombre de répétitions ( 3 feuilles et 3 fruits sur chaque pied), les sites étant le traitement principal et les sous-variétés le traitement secondaire. L'évaluation des descripteurs morphologiques a été respectivement faite sur 768 feuilles et fruits. Les critères de différenciation paysanne étaient liés à l'observation (couleur, forme, etc.) des arbres, feuilles et fruits. Les descripteurs de l'accessibilité à une meilleure luminosité de l'arbre, du rendement et du développement végétatif pourront servir de références lors de la domestication de la plante. Les sous-variétés 1, 2 et 3 étaient hautement significatives, de par leur valeur d'usage ethnobotanique total, supérieure à 3 parmi les 4 recensées. Des études moléculaires utilisant les ADN génomiques (chloroplastique et ribosomal) permettront de tirer une conclusion définitive quant à l'origine environnementale ou moléculaire de la variabilité observée au sein de la plante dans cette zone.

Mots-clés : ressource locale, plante multifonctionnelle, conservation de la diversité biologique, savoir endogène, descripteurs morphologiques, valeur d'usage ethnobotanique, facteur de consensus des informateurs, plan factoriel, Syzygium guineense var. macrocarpum, hautes savanes guinéennes, Adamaoua, Cameroun.

\section{Ethnobotany and morphological variability of Syzygium guineense var. macrocarpum in the upland Guinean savannah of Adamaoua (Cameroon)}

\section{ABSTRACT}

The morphological variability of the leaves and fruits of many local multifunctional plant species is a topic of particular concern to African researchers, all the more so in view of the threat of extinction of certain plants that still exist in the natural environment. One such species found in the upland savannah agroecological zone of Adamaoua in Cameroon is Syzygium guineense var. macrocarpum (Engl.) F. White, an endemic species of Myrtaceae. The problem has arisen of which sub-variety to choose for domestication purposes. Ethnobotany, because it investigates the relationships between people and plants over time, can provide essential information on local knowledge and preferences. Our aims were also to identify, describe and determine morphological descriptors (qualitative and quantitative) and sub-varieties of the plant. For this purpose, we interviewed 218 men and women from nine different ethnic groups and selected 256 trees belonging to the plant's sub-varieties growing in $100 \mathrm{~m} \times 100 \mathrm{~m}$ quadrats. followed $\mathrm{A}$ factorial design $(8 \times 4 \times 8 \times 3)$ was chosen for the experimental set-up: $8 \times 4$ for the experimental unit; 8 for the number of study sites; 4 for the number of sub-varieties; 3 for the number of repetitions ( 3 leaves and 3 fruits on each stem), with primary data analysis for the sites and secondary analysis for the sub-varieties. The morphological descriptors were assessed for 768 leaves and fruits. Criteria for differentiation by peasant families were based on observations (colour, shape, etc.) of the trees, leaves and fruits. The descriptors for tree accessibility to better light conditions, yield and vegetative growth could be used as references in attempts to domesticate the plant. Sub-varieties 1, 2 and 3 were highly significant, with a total ethnobotanical use value of more than 3 out of the 4 identified. Molecular studies of genomic DNA (from chloroplasts and ribosomes) will help to draw final conclusions as to the environmental or molecular origin of the variability observed in this plant in the study zone.

Keywords: local resource, multifunctional plant species, conservation of biological diversity, endogenous knowledge, morphological descriptors, ethnobotanical use value, consensus among informants, factorial design, Syzygium guineense var. macrocarpum, upland Guinean savannah, Adamawa, Cameroon.

\section{Estudio etnobotánico y variabilidad morfológica de Syzygium guineense var. macrocarpum en las altas sabanas guineanas de Adamaoua (Camerún)}

\section{RESUMEN}

La problemática de la variabilidad morfológica de las hojas y frutos de numerosas plantas locales multifuncionales preocupa a los investigadores africanos. A ello se añade el riesgo de desaparición de algunas de estas plantas que viven todavía de forma natural. En la zona agroecológica de las altas sabanas guineanas de Adamaoua, en Camerún, se encuentra Syzygium guineense var. macrocarpum (Engl.) F. White, una mirtácea endémica. Para su domesticación se ha planteado la cuestión de la elección de las subvariedades que se quieren mantener. Como la etnobotánica es la relación entre el hombre y las plantas en un medio dado a través del tiempo, es indispensable tener en cuenta el saber y las preferencias locales. Asimismo, el estudio pretendía identificar, describir y determinar los descriptores morfológicos (cualitativos y cuantitativos) y las subvariedades de la planta. Las personas encuestadas fueron 218 hombres y mujeres pertenecientes a nueve etnias diferentes. En cuadrados de $100 \mathrm{~m} \times 100 \mathrm{~m}$, se seleccionaron 256 árboles de las subvariedades de la planta. El dispositivo experimental era un plano factorial $(8 \times 4 \times 8 \times 3): 8 \times 4$ para la unidad experimental; 8 para el número de zonas de estudio; 4 para el número de subvariedades; 3 para el número de repeticiones ( 3 hojas y 3 frutos en cada pie). Las zonas fueron el estudio principal y las subvariedades, el secundario. La evaluación de los descriptores morfológicos se realizó en 768 hojas y frutos. Los criterios de diferenciación agrícola se relacionaron con la observación (color, forma, etc.) de los árboles, hojas y frutos. Los descriptores de la accesibilidad a una mejor luminosidad del árbol, de rendimiento y de desarrollo vegetativo podrán servir de referencia durante la domesticación de la planta. Las subvariedades 1,2 y 3 resultaron altamente significativas por su valor de uso etnobotánico total, superior en tres de entre las cuatro subvariedades censadas. Los estudios moleculares que utilizan los ADN genómicos (cloroplástico y ribosómico) permitirán obtener una conclusión definitiva en cuanto al origen medioambiental y molecular de la variabilidad observada en la planta en esta zona.

Palabras clave: recurso local, planta multifuncional, conservación de la diversidad biológica, saber endógeno, descriptores morfológicos, valor de uso etnobotánico, factor de consenso de los informadores, plan factorial, Syzygium guineense var. macrocarpum, altas sabanas guineanas, Adamawa, Camerún.
Doi : $10.19182 / \mathrm{bft} 2020.343 . a 31852$

Droit d'auteur (c) 2020, Bois et Forêts des Tropiques @ C Cirad Date de publication : 28 février 2020 


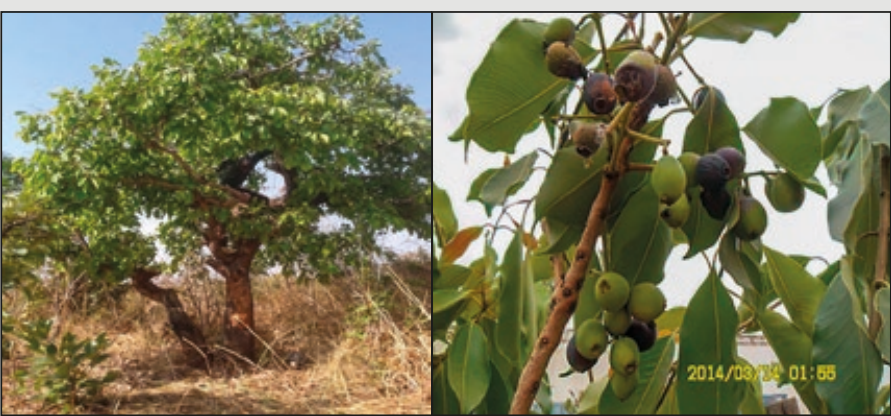

Photos 1.

Pied de Syzygium guineense (Willd.) DC. var. macrocarpum (Engl.) F. White (à gauche) et détails des feuilles et des fruits (à droite). Photo G. M. Lamy.

Photos 1.

A specimen of Syzygium guineense (Willd.) DC. var. macrocarpum (Engl.) F. White (left) and detail of flowers and fruits (right).

Photo G. M. Lamy.

Fotos 1.

Pie de Syzygium guineense (Willd.) DC. var. macrocarpum (Engl.) F. White (a la izquierda) y detalles de las hojas y de los frutos (a la derecha).

Foto G. M. Lamy.

\section{Publications}

Lamy Lamy G. M., Ibrahima A., Ndjonka D., Mapongmetsem P. M., 2018. Étude ethnobotanique des sous-variétés de Syzygium guineense (Will.) DC. var. macrocarpum (Engl.) F. White dans les Hautes Savanes Guinéennes (Adamaoua, Cameroun). International Journal of Biological and Chemical Sciences, 12 (4) : 1636-1649. https://dx.doi.org/10.4314/ijbcs.v12i4.11

Lamy Lamy. G. M., Ndjonka D., Ibrahima A., Mapongmetsem P. M., 2019. Contribution of ethnobotanical results in the process of domestication of an agroforestry plant with morphological variability (Adamawa, Cameroon). Ethnobotany Research and Applications, 18 (12): 1-14. http://dx.doi.org/10.17348/era.18.12.1-14

Grade et diplôme : Docteur (PhD) en Biologie végétale, spécialité Botanique et Écologie.

Université : Université de Ngaoundéré, Cameroun.

Date de soutenance : vendredi 17 mai 2019.

\section{Composition du jury}

Direction : Adamou Ibrahima (Maître de Conférences, Université de Ngaoundéré, Laboratoire de Biodiversité et de Développement durable), Dieudonné NDJONKA (Professeur, Université de Ngaoundéré, Cameroun), Pierre Marie MAPongmetsem (Professeur, Université de Ngaoundéré, Cameroun).

Membres : Fernand-Nestor Tchuenguem Fohouo (Professeur, Faculté des Sciences, Université de Ngaoundéré, Cameroun), Clautilde Meguenı (Maître de Conférences, Université de Ngaoundéré, Cameroun), Albert NgAKou (Professeur, Université de Ngaoundéré, Cameroun), Louis ZAPFACK (Maître de Conférences, Université de Yaoundé I, Cameroun), TchobSALA (Maître de Conférences, Université de Maroua, Cameroun).

Langue de rédaction : français.

Accès au manuscrit : https://www.researchgate.net/profile/ georges lamy lamy

Contact : geomaxlamy@gmail.com

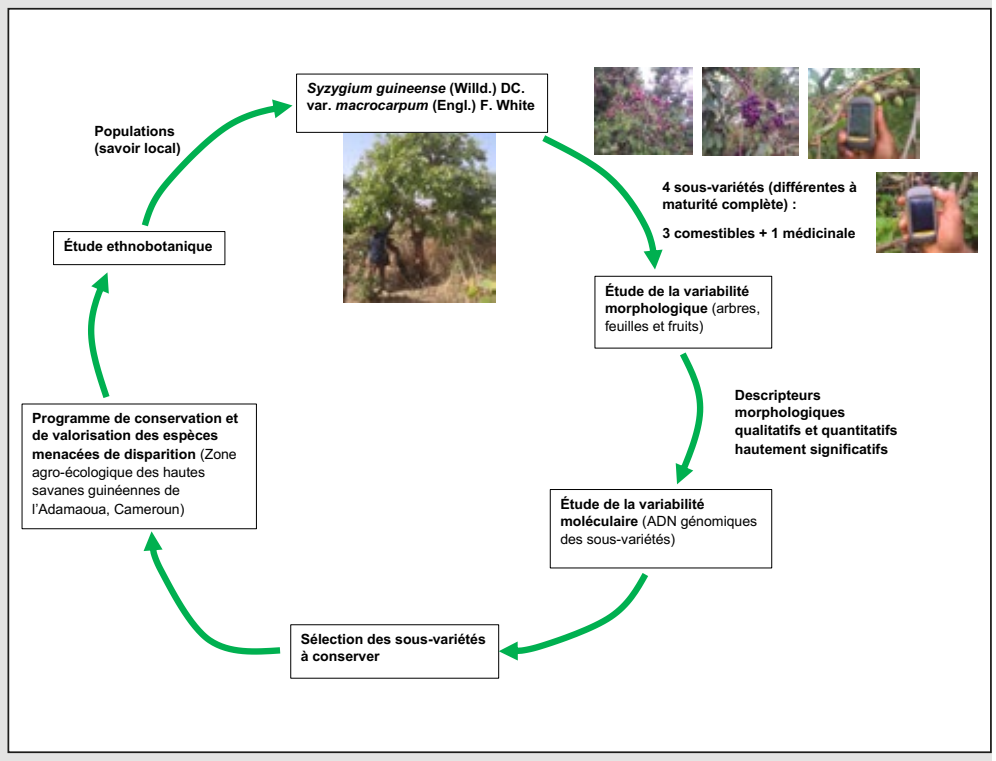

Figure 1.

Processus de conservation des sous-variétés de Syzygium guineense (Willd.) DC. var. macrocarpum (Engl.) F. White.

Figure 1.

Process for conserving sub-varieties of Syzygium guineense (Willd.) DC. var. macrocarpum (Engl.) F. White. Translation of the captions of the figure: 4 sous-variétés (différentes à maturité complète) :

3 comestibles +1 médicinale: 4 sub-varieties (differing when fully mature): 3 edible +1 medicinal; Étude de la variabilité morphologique (arbres, feuilles et fruits): Study of morphological variability (trees, leaves and fruits); Descripteurs morphologiques qualitatifs et quantitatifs hautement significatifs: Highly significant qualitative and quantitative morphological descriptors; Étude de la variabilité moléculaire (ADN génomiques des sous-variétés): Study of molecular variability (genomic DNA of sub-varieties); Sélection des sous-variétés à conserver: Selection of sub-varieties for conservation; Programme de conservation et de valorisation des espèces menacées de disparition (Zone agro-écologique des hautes savanes guinéennes de l'Adamaoua Cameroun): Programme for conservation and use of endangered species (Adamaoua upland Guinean savannah agro-ecological zone, Cameroon); Étude ethnobotanique: Ethnobotanical study; Populations (savoir local): Populations (local knowledge).

Figura 1.

Proceso de conservación de subvariedades de Syzygium guineense (Willd.) DC. var. macrocarpum (Engl.) F. White. Traducción de las leyendas de la figura: 4 sous-variétés (différentes à maturité complète) : 3 comestibles +1 médicinale: 4 subvariedades (diferentes en la completa madurez): 3 comestibles +1 medicinal; Étude de la variabilité morphologique (arbres, feuilles et fruits): Estudio de la variabilidad morfológica (árboles, hojas y frutos); Descripteurs morphologiques qualitatifs et quantitatifs hautement significatifs: Descriptores morfológicos cualitativos y cuantitativos altamente significativos; Étude de la variabilité moléculaire (ADN génomiques des sousvariétés): Estudio de la variabilidad molecular (ADN genómico de las subvariedades); Sélection des sous-variétés à conserver: Selección de las subvariedades a conservar; Programme de conservation et de valorisation des espèces menacées de disparition (Zone agro-écologique des hautes savanes quinéennes de l'Adamaoua, Cameroun): Programa de conservación y de valorización de las especies amenazadas de desaparición (zona agroecológica de las altas sabanas guineanas de Adamaoua, Camerún); Étude ethnobotanique: Estudio etnobotánico; Populations (savoir local): Poblaciones (saber local). 